\title{
Comorbid anxiety and depression: bête noire or quick fix? ${ }^{\dagger}$
}

Carmen Andreescu and Eric J. Lenze

\section{Summary}

The common territory shared by anxiety and depression has always been a contentious subject. Research in favour of anxious depression as a potentially treatment-relevant subtype has been limited by diagnostic dilemmas and crude measurement. The most recent evidence from genetics, neuropeptide systems and functional neuroimaging suggests a valid diagnostic construct.

\section{Declaration of interest}

E.J.L. has received research support from Forest, Johnson \& Johnson and Roche, as well as (medications only) Pfizer and Bristol-Myers Squibb.
Carmen Andreescu (pictured) is Assistant Professor of Psychiatry at Western Psychiatric Institute and Clinic and conducts research in the neuroimaging of mood and anxiety disorders. Eric Lenze is Professor of Psychiatry at Washington University and conducts treatment studies in depression and anxiety.

The peripatetic journey of psychiatric nosology has triggered a wealth of reactions, from militant antipsychiatry to philosophical anchorage, ${ }^{1}$ from warnings of 'brainlessness' and 'mindlessness' drifts to derision of high-tech creeds. ${ }^{2}$ Psychiatrists have been often portrayed as either obsessional splitters or narcissistic wizards, spreading imaginary epidemics to quench an Adlerian thirst for power or just to get rich.

More sympathetic attempts to understand these vacillations describe models such as epistemic iteration, according to which successive stages of knowledge build increasingly accurate estimations of a diagnostic model. ${ }^{3}$ This would involve a stable, objective model - in other words, an entity that exists 'out in the world' but for now eludes our ability to define it. That brings us to the crux of this editorial.

Comorbidity between anxiety and depression has received constant attention from generations of researchers. But does anxious depression exist 'out there'? Any clinician would say yes, as they encounter and treat the mixed version more often than pure depression. However, as dichotomisers have ruled the DSM for some time, ${ }^{1}$ the US psychiatrist will have to write two diagnoses to accommodate the symptomatology to the nosology.

Several theoretical models argued that a diagnosis of 'anxious depression' is a quick fix, an artefact forced upon naturally dimensional psychopathologies by the dominant neoKraepelinian paradigm. Others support the view of two different entities sharing some common psychopathological territory or representing, in their mixed state, the stable, deepest core of neurotic symptoms. ${ }^{4}$ Models like the tripartite model of Clark \& Watson rely on psychological constructs such as positive affectivity, physiological hyperarousal and negative affectivity. ${ }^{5}$ Empirical research has supported the tripartite model's utility, especially with regard to the two dimensions that separate depression and anxiety (low positive affect and high physiological hyperarousal, respectively), which have proven to be orthogonal. The comorbidity is explained almost exclusively by an increase

\footnotetext{
${ }^{\dagger}$ See pp. 210-215, this issue.
}

in negative affect in both conditions, which links anxiety and depression with constructs such as neuroticism. Other models, such as the approach-withdrawal model or the valence-arousal model, rely on affective styles stemming from the interaction of motivation and emotion, as well as on the neural circuits assumed to underlie these constructs. ${ }^{6}$ The approach system (positive affect and reward response) and the withdrawal system (avoidance and negative affect) involve overlapping, yet dissociable neural circuits: left prefrontal cortex and the basal ganglia for the approach system, right prefrontal cortex for the withdrawal system, with the amygdala playing a crucial role in both systems. ${ }^{6}$ These models propose various permutations among the basic factors as the source of comorbidity, but as a rule, they advance endophenotypes designed to increase the diagnostic validity.

More recently, bipolar vulnerability has also been suggested as a source of comorbidity. The study by Coryell and colleagues ${ }^{7}$ in this issue supports the prognostic importance of anxiety symptoms in the long-term outcome of both unipolar and bipolar depression.

Further insights into the sources of comorbidity have come from gene-environment interaction studies. These have shown that genetic risk factors for major depression and generalised anxiety are strongly correlated, but that the majority of the genetic covariance between the two disorders results from factors not shared with neuroticism (which accounted for only about 25\% of this correlation). ${ }^{8}$ Such results contradict the architecture of the tripartite model, in which negative affect is the intermediate phenotype of comorbid anxiety-depression. In addition, neuroticism-independent genetic factors seem to significantly increase the risk for major depression, generalised anxiety disorder and panic disorder, showing that there is substantial, but not complete, overlap between the genetic factors that influence individual variation in neuroticism and those that increase liability for both depression and anxiety.

As epistemic iteration requires building on incremental knowledge, the construct of comorbid depression and anxiety is currently being deliberated not through sophisticated psychological models, but through progress in the biological realms of receptor changes, neuropeptide systems alterations, dysregulation in intracellular signalling, changes in gene sequence or expression, or alteration in brain circuits. ${ }^{9}$ How relevant are these advances to the pathophysiology of anxious depression? A brief overview is required.

Serotonin plays an important modulatory role in emotion, motivation and cognition, and its dysfunction contributes to 
many disorders, including mood and anxiety disorders, psychosis and substance misuse. Serotonin transporter knockout rodents have been extensively characterised in well-validated tests for anxiety- and depression-like behaviour. However, depression and anxiety-like symptoms are less robust in these animal models, suggesting that the monoaminergic dysregulation is most likely intermingled with dysregulation in other systems such as the glutamatergic or peptidergic systems, in particular neuropeptide Y (NPY) and vasopressin.9 "The importance of vasopressin, corticotropin-releasing hormone, oxytocin, prolactin, neuropeptide $Y$ and neuropeptide $S$ as neuromodulators of emotionality is becoming increasingly apparent. Differences in these peptides' behaviour in depression $v$. anxiety models are frequently reported. ${ }^{9}$ Thus, specific agonists of the NPY1 receptor are purely anxiolytic, whereas NPY2 antagonists have anxiolytic and antidepressant potential; urocortin 1 has no effect in depressive models, whereas it has anxiogenic properties; oxytocin has been extensively studied for its anxiolytic effect, whereas its antidepressant effect is still unclear. So far, there are no studies addressing neuropeptide changes in comorbid anxious depression, so we cannot make inferences regarding their role in supporting a distinct diagnosis of anxious depression.

One relevant neurotrophin that has been connected to comorbid anxious depression is the brain-derived neurotrophic factor (BDNF). Recent data showed that the BDNF Val66Met allele was significantly more abundant in individuals with comorbid anxious depression than in individuals with pure depression or pure anxiety. Proinflammatory cytokines (interferon-alpha, interleukin-2, interleukin-1-beta, interferongamma) have been implicated in the pathophysiology of mood disorders, through their influence on monoaminergic metabolism and the hypothalamic-pituitary-adrenocortical (HPA) axis, but again, there are no studies addressing their role in anxious depression. This brings us to the most studied system - the HPA axis. Clinical and preclinical studies have reported HPA-axis dysregulation in mood and anxiety disorders, with higher cortisol levels in comorbid anxious depression than in pure major depressive disorder or pure generalised anxiety disorder. The association of HPA axis and the tripartite model of Clark \& Watson $^{5}$ has shown that morning cortisol was not linked to DSM-IV diagnoses of anxiety disorders or major depressive disorder, but to specific symptoms such as anhedonia, worry and negative affect, thus making an argument for a dimensional diagnostic model.

Although neuroimaging studies pertinent to either depression or anxiety have flooded MEDLINE in the past two decades and transformed the amygdala into a star, a surprisingly small number of studies explored the neural markers of anxiety-depression comorbidity. ${ }^{10}$ Anxiety-depression comorbidity has been characterised by more right than left hemispheric anterior activity in people with major depressive disorder, consistent with a key role of the right prefrontal cortex in anxiety disorders. ${ }^{6}$ Sustained activation in the dorsal anterior cingulate cortex has been described as a marker of anxiety superimposed on depression, results confirmed by a voxel-based morphometry study indicating that reduced volume of dorsal anterior cingulate cortex is a non-specific effect of comorbid anxiety and depression. Moreover, the connectivity patterns in the default-mode network in late-life depression are modified by the presence of increased anxiety symptoms. ${ }^{10}$ The functional neuroimaging experiments have still to move the field forward clinically, to offer sensitive and specific biomarkers of diagnostic and treatment response, but the few results available suggest that comorbid anxious depression leaves a different neural imprint than pure depression or pure anxiety.
To return to the epistemic iteration model, it seems we generate increasingly accurate estimations of the biological features of 'out-in-the-world' anxious depression, but the asymptotic nature of the process does not allow for fast gratification. The overall direction of the most recent biological findings points towards a valid comorbid entity, one that has been for a long time a nosological bête noir owing to its complexity. The current failure to allow the diagnosis of mixed anxiety-depression (subthreshold major depressive disorder plus subthreshold anxiety disorder) has notable treatment consequences: practitioners may not provide optimal pharmacotherapy or psychotherapy (such as simple-to-deliver internet cognitive-behavioural therapy ${ }^{11}$ ), or even fail to detect or treat the symptoms as they do not fall in the prescribed DSM category. ${ }^{12}$

We expect eventual applications from neuroscience and genetics to revolutionise the diagnosis and treatment of mental disorders. Current efforts, including those listed above, remind us though that we tackle the 'most complexly organized structure in the universe $[\ldots]$ and the number of possible permutations and combinations of brain activity, in other words the numbers of brain states, exceeds the number of elementary particles in the known universe. ${ }^{13}$

Maybe attempts to achieve DSM validity, especially in contentious areas like comorbid anxious depression, will take more iterations, but some of the dismissive approaches reviewed in the first paragraph of this editorial remind us of a story about the late Francis Crick: 'All this stuff on the brain is interesting, Dr Crick', a lady asked him during a fund-raiser, 'but can you name any one single discovery in the last two decades that has really important implications?' 'Well, my dear', replied Crick, 'one thing we have now learnt is that the brain is really plastic. ${ }^{14}$

Carmen Andreescu, MD, Department of Psychiatry, University of Pittsburgh Pittsburgh, Pennsylvania, USA; Eric Lenze, MD, Department of Psychiatry, Washington University, Saint Louis, Missouri, USA

Correspondence: Carmen Andreescu, Western Psychiatric Institute and Clinic, 3811 O'Hara Street, Pittsburgh, PA 15213, USA. Email: Andreescuc@upmc.edu

First received 19 Jul 2011, final revision 28 Sep 2011, accepted 19 Dec 2011

\section{Acknowledgements}

C.A. is supported in part by $\mathrm{NHH}$ grants $\mathrm{K} 23 \mathrm{MH} 08668$, R01 MH070547, the National Alliance for Research on Schizophrenia and Depression (NARSAD) Young Investigator Award. The authors are grateful to Drs Mary Ganguli, Howard Aizenstein, Ydwine Zanstra and Peter Tyrer for their comments and suggestions.

\section{References}

1 Frances A, Mack AH, First MB, Widiger TA, Ross R, Forman L, et al. DMS-IV meets philosophy. J Med Philos 1994; 19: 207-18.

2 Eisenberg L. Mindlessness and brainlessness in psychiatry. $\mathrm{Br} J$ Psychiatry 1986; 148: 497-508.

3 Kendler KS. An historical framework for psychiatric nosology. Psychol Med 2009; 39: 1935-41.

4 Tyrer $\mathrm{P}$, Seivewright $\mathrm{H}$, Johnson $\mathrm{T}$. The core elements of neurosis: mixed anxiety-depression (cothymia) and personality disorder. J Pers Disord 2003; 17: 129-38.

5 Clark LA, Watson D. Tripartite model of anxiety and depression: psychometric evidence and taxonomic implications. J Abnorm Psychol 1991; 100: $316-36$.

6 Davidson RJ. Affective style and affective disorders: perspectives from affective neuroscience. Cogn Emot 1998; 12: 307-30.

7 Coryell W, Fiedorowicz JG, Solomon D, Leon A, Rice JP, Keller MB. Effects of anxiety on the long-term course of depressive disorders. Br J Psychiatry 2012; 200: 210-5. 
8 Hettema JM, Neale MC, Myers JM, Prescott CA, Kendler KS. A populationbased twin study of the relationship between neuroticism and internalizing disorders. Am J Psychiatry 2006; 163: 857-64.

9 Neumann ID, Wegener G, Homberg JR, Cohen H, Slattery DA, Zohar J, et al. Animal models of depression and anxiety: what do they tell us about human condition? Prog Neuropsychopharmacol Biol Psychiatry 2011; 35: 1357-75.

10 Andreescu C, Wu M, Butters MA, Figurski J, Reynolds 3rd CF, Aizenstein HJ. The default mode network in late-life anxious depression. Am J Geriatr Psychiatry 2011; 19: 980-3.
11 Titov N, Dear BF, Schwencke G, Andrews G, Johnston L, Craske MG, et al. Transdiagnostic internet treatment for anxiety and depression: a randomised controlled trial. Behav Res Ther 2011; 49: 441-52.

12 Goldberg D. Should our major classifications of mental disorders be revised? Br J Psychiatry 2010; 196: 255-6.

13 Ramachandran V, Blakeslee, S, Sacks, O. Phantoms in the Brain: Probing the Mysteries of the Human Mind. Harper Perennial, 1999.

14 Ramachandran V. The astonishing Francis Crick. Edge 2004; 147: 1 (http:// www.edge.org/3rd_culture/crick04/crick04_index.html).

\section{psychiatry in 19th-century literature}

\section{Dostoevsky and psychoanalysis: The Eternal Husband (1870) by Fyodor Dostoevsky (1821-1881)}

\author{
Ruth Yvonne Pavlovic and Alexandar Mido Pavlovic
}

Dostoevsky, the great 19th-century Russian author, was the son of a Muscovite doctor. Raised near a lunatic asylum, he suffered from epilepsy, neuroticism and gambling addiction. His writings (The Brothers Karamazov, The Idiot and Notes from Underground) reveal rich portrayals of psychiatric cases, including epilepsy, hysteria, dementia and psychopathy, and his psychological insights are remarkable in world literature. His characterisation of unconscious psychological motivation prefigures terms later described by the psychoanalytic movement.

Dostoevsky's novel The Eternal Husband appeared in 1870, when Sigmund Freud was just 14. It portrays defence mechanisms now known as repression, reaction formation, displacement and rationalisation, as well as unconscious material surfacing in dreams and impulses. The tale depicts a cuckold (Pavlovitch) who after his wife's death discovers her infidelity. He tracks down Velchaninov, his wife's former lover. Velchaninov does not recognise him; his memories of the affair are initially repressed. However, psychic unease ferments in his dreams. In 1895 - 4 years after Dostoevsky's death - Freud coined the term 'repression' to refer to the ego defence mechanism of excluding undesired impulses from consciousness. By 1900, Freud's classic text acknowledged dream interpretation as the 'royal road to . . . the unconscious'.

Pavlovitch is unaware of his purpose in compulsively seeking velchaninov. Overtly, he embraces him, drinking with him and declaring his love. However, unconscious hatred emerges one night in his impulsive attempt to stab him. Velchaninov concludes, 'love that comes from hate is strongest of all', indicating Pavlovitch's friendliness was a defence Freud later termed 'reaction formation'. Pavlovitch, discovering his beloved daughter, Liza was sired by Velchaninov, displaces hatred for Velchaninov onto her with cruel torment. Velchaninov senses he himself is driven by unexplained forces, but rationalises his actions.

Not until 1926 (Inhibitions, Symptoms and Anxiety) did Freud reformulate repression as one among many defences, naming other mechanisms (reaction formation, displacement and rationalisation) which operate when repressed material threatens to return to consciousness. Dostoevsky certainly anticipated psychoanalytic concepts; but did he influence the developing psychoanalytic movement? Freud was familiar with Dostoevsky's works. The Eternal Husband appeared in German circa 1900 and Freud's Viennese acquaintances, Rene Fulop-Miller and Friedrich Eckstein, collected Dostoevsky's supplementary manuscripts for German publication, approaching Freud for comment. Freud commenced Dostoyevsky and Parricide (an introduction to drafts of The Brothers Karamazov) in 1926, the same year he revised his theory of defences.

Analysing Dostoevsky's personality from his fictional characterisation, Freud detected Oedipal and sexual conflicts behind the writer's 'hysterical epilepsy' and gambling. Of 'Dostoevsky the psychologist', Freud complained his 'insight was so restricted to abnormal mental life. . . all he really knew were crude instinctual desire, masochistic subjection and loving out of pity'. However, Freud bowed to his artistry: 'before the . . . creative artist, analysis must lay down its arms'. He pronounced The Brothers Karamazov the 'most magnificent novel ever written'.

Some Freudian case histories (Rat Man, 1909) unfold artistically and psychologically like the plot of The Eternal Husband, whereby narration of seemingly inexplicable impulses builds to a concluding denouement by the protagonist/analyst, rendering unconscious processes explicit. Both formats revolve around the centrality of unconscious motivation and its exposure. 